\title{
ABBREVIATIONS
}

\section{BOOKS AND Journals}

AAA
$A B S A$
$A H B$
$A H R$
$A 7 A H$
$A 7 P b$
AncW
Ant. Class.
BHM
BICS
CAH
CCC
Cbron. d'Ég.
CIL
Class. et Med.
Cf
CPb
CQ
CR
Daremberg-Saglio
D-K

Athens Annals of Archaeology

Annual of the British School at Athens

Ancient History Bulletin

American Historical Review

American fournal of Ancient History

American Fournal of Philology

Ancient World

L'Antiquité classique

Bulletin of the History of Medicine

Bulletin of the Institute of Classical Studies, University of London

Cambridge Ancient History

Civiltà classica e Cristiana

Chronique d'Égypte.

Corpus inscriptionum Latinarum (Berlin, I863-)

Classica et medievalia

Classical fournal

Classical Philology

Classical Quarterly

Classical Review

C. Daremberg and E. Saglio, Dictionnaire des

Antiquités grecques et romaines (Paris, I877-1919)

H. Diels and W. Kranz, Die Fragmente der

Vorsokratiker, I2th ed., 3 vols. (Dublin and Zürich, 1966)

EMC/CV Échos du Monde Classique/Classical Views

$\mathrm{FGrH}$

F. Jacoby, Fragmente der griechischen Historiker

(Berlin and Leiden, 1923-58) 
FHG

GHI

GMPT

Gomme-Andrewes-

Dover

GRBYS

Hippocr.

HSCP

$H T h R$

$I G$

$7 A W$

$7 E A$

7HS

K-R-S

LIMC

L-P

Meiggs-Lewis

Mus. Helv.

$O C D^{2,3}$

OGIS

$P D M$

$P G M$
K. and Th. Müller, Fragmenta Historicorum Graecorum (Paris, $184 \mathrm{I}^{-85}$ )

M. N. Tod, A Selection of Greek Historical Inscriptions, vol. 2, From 403-323 B.C. (Oxford, 1948)

H. D. Betz, The Greek Magical Papyri in Translation, Including the Demotic Spells, vol. I, Texts, $2 \mathrm{~d}$ ed. (Chicago, 1992)

A. W. Gomme, A. Andrewes, and K. J. Dover, A Historical Commentary on Thucydides, vol. 4 (Oxford, 1970)

Greek, Roman and Byzantine Studies

Hippocratic Corpus

Harvard Studies in Classical Philology

Harvard Theological Review

Inscriptiones Graecae (Berlin, 1873-)

[Bursian's] Jabresbericht über die Fortschritte der

Klassichen Altertumswissenschaft (Berlin,

I873-1944/55)

Fournal of Egyptian Archaeology

Fournal of Hellenic Studies

G. S. Kirk, J. E. Raven, and M. Schofield, The

Presocratic Philosophers: A Critical History with a

Selection of Texts, 2d ed. (Cambridge, 1983)

Lexicon Iconographicum Mythologiae Classicae, var. edd.

(Zürich and Munich), vol. I: 1981; vol. 2: 1984;

vol. 3: 1986; vol. 4: 1988; vol. 5: 1990; vol. 6: 1992;

vol. 7: 1994

E. Lobel and D. L. Page, Poetarum Lesbiorum

Fragmenta (Oxford, 1955)

R. Meiggs and D. M. Lewis, Greek Historical

Inscriptions to the End of the Fifth Century B.C.

(Oxford, 1969)

Museum Helveticum

The Oxford Classical Dictionary (Oxford, 2d ed.: 1970;

3d ed.: 1996)

W. Dittenberger, Orientis Graecae Inscriptiones

Selectae, 2 vols. (Leipzig, 1903-5)

Papyri demoticae magicae (see also GMPT)

K. Preisendanz, Papyri Graecae Magicae: Die 
Pbilol.

griechischen Zauberpapyri, 2 vols., 2d ed. (Stuttgart,

$P M G$

PWK

$R E G$

$R b M$

$S L G$

Suppl. Mag.

SVF

$T A P h A$

YClS

$Z P E$

Aesch.

Aeschin.

Alciphr.

Amm. Marc.

Andoc.

Ant. Lib.

$A P$
1973-74)

Philologus

D. L. Page, Poetae Melici Graeci (Oxford, 1962)

A. Pauly, G. Wissowa, W. Kroll, K. Ziegler, RealEncyclopädie der classischen Altertumswissenschaft, 83 vols. (Stuttgart, I894-1980)

Revue des études grecques

Rheinisches Museum

D. L. Page, Supplementum Lyricis Graecis (Oxford, I974)

Supplementum Magicum

J. von Arnim, Stoicorum Veterum Fragmenta, 4 vols.

(Leipzig, 1902-24)

Transactions and Proceedings of the American

Philological Association

Yale Classical Studies

Zeitschrift für Papyrologie und Epigraphik

\section{Ancient Sources}

Claudius Aelianus, A.D. I65/70-230/5

Hellenized Roman essayist

$$
V H=\text { Varia Historia }
$$

Aeschylus, 525/4-456 в.с.

Athenian tragedian

$$
\text { Pers. }=\text { The Persians }
$$

Aeschines, c. 397-c. 322 B.c.

Athenian orator

Alciphron, ?2d-3d century A.D.

Greek sophist and writer

$$
\text { Ep. Amat. = Epistulae Amatoriae }
$$

Ammianus Marcellinus, c. A.D. 330-395

Roman historian

Andocides, c. 440-c. 390 B.c.

Athenian aristocrat and politician

Antoninus Liberalis, ?2d century A.D.

Mythographer

Anthologia Palatina 
Apollod.

App.

Ap. Rhod.

Arat.

Arist.

Aristoph.

Arrian

Athen.
Apollodorus, c. I80-c. IIо B.c.

Mythographer Epit. $=$ Epitome

Appianos [Appian] of Alexandria, $2 \mathrm{~d}$ century A.D.

Greek historian

$$
\begin{aligned}
& \text { B.C. }=\text { Bella Civilia } \\
& \text { Mithr. = Mitbridaticum [Bellum] }
\end{aligned}
$$

Apollonius Rhodius, c. 300-c. 23 в в.c.

Alexandrian epic poet

$$
\text { Arg. = Argonautika }
$$

Aratus of Soli, c. 315-240 B.c.

Didactic poet

$$
\text { Phaen. }=\text { Phaenomena }
$$

Aristotle of Stagira, 384-322 в.c.

Greek philosopher

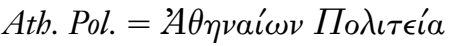

$$
\begin{aligned}
& \text { Eth. Nic. = Ethica Nicomachea } \\
& \text { Met. }=\text { Meteorologica } \\
& \text { Metaph. }=\text { Metaphysica } \\
& \text { Oecon. }=\text { Oeconomica } \\
& \text { Poet. }=\text { Poetica } \\
& \text { Pol. }=\text { Politica } \\
& \text { Probl. }=\text { Problemata } \\
& \text { Rhet. }=\text { Rhetorica }
\end{aligned}
$$

Aristophanes, c. $45^{\circ}$-c. 385 в.c.

Athenian comic playwright

$$
\begin{aligned}
& \text { Ach. }=\text { Acharnians } \\
& \text { Eccles. }=\text { Ecclesiazusae } \\
& \text { Kn. }=\text { Knights } \\
& \text { Plut. }=\text { Plutus } \\
& \text { Thesm. = Thesmophoriazusae }
\end{aligned}
$$

L. Flavius Arrianus, c. A.D. 86-I6o

Romanized Greek historian

$$
\begin{aligned}
& \text { Anab. = Anabasis } \\
& \tau \dot{\alpha} \mu \epsilon \tau \dot{\alpha} ' A \lambda \epsilon^{\prime} \xi=\tau \dot{\alpha} \mu \epsilon \tau \grave{\alpha} ' A \lambda \lambda^{\prime} \xi \alpha \nu \delta \rho o \nu \\
& \text { (Affairs after Alexander) }
\end{aligned}
$$

Athenaeus of Naucratis, fl. c. A.D. 200

Belle-lettrist

Deipnos. $=$ Deipnosophistae 
Aug.

Caes.

Callim.

Cato

Catull.

Cels.

Cic.

Clem. Alex.

Curt.

Dem.

dieg.

Dio Cass.

Dio Chrys.
St. Augustine of Hippo, A.D. 354-430

Christian apologist

Civ. Dei $=$ Civitas Dei

C. Julius Caesar, ıoo-44 B.c.

Roman statesman and historian

Bell. Alex. = Bellum Alexandrinum

Callimachus of Cyrene, ?310-?240 B.C.

Alexandrian poet and scholar

$$
H .=H y m n
$$

M. Porcius Cato, 234-I49 B.c.

Roman statesman and writer

$$
\text { De Agr: = De Agri Cultura }
$$

C. Valerius Catullus, c. 84-c. 54 B.c.

Roman lyric and elegiac poet

A. Cornelius Celsus, early ist cent. A.D.

Medical writer

$$
\text { De Med. }=\text { De Medicina }
$$

M. Tullius Cicero, ıo6-43 в.c.

Roman orator and writer

Att. = Epistulae ad Atticum

De Div. = De Divinatione

De Fin. = De Finibus Bonorum et Malorum

De Rep. = De Republica

Fam. = Epistulae ad Familiares

$N D=$ De Natura Deorum

T. Flavius Clemens Alexandrinus [Clement of

Alexandria], c. A.D. I5O-c. 215

Christian theologian

Protrept. $=$ Protrepticus

Strom. $=$ Stromateis

Q. Curtius Rufus, fl. c. A.D. 50

Roman historian

Demosthenes, 384-322 в.c.

Athenian orator

diegesis [narrative résumé]

L.(?) Cassius Dio, c. A.D. I64-c. 230

Romanized Greek historian

Dio Chrysostomus [Cocceianus],

c. A.D. $40 / 5 \mathrm{O}-$ c. IIO 
Diod. Sic.

Diog. Laert.

Dion. Hal.

Eur.

Euseb.

Eustath.

Galen

Hdt.

Herod.
Greek orator

Diodorus Siculus of Agyrium, ist century в.c.

Greek historian

Diogenes Laertius, ?early 3 d century A.D.

Greek biographer

Dionysius Halicarnassensis, c. 5 O B.C.-c. A.D. 20

Greek critic and historian

Ant. Rom. $=$ Antiquitates Romanae

Ep. ad Pomp. = Epistula ad Gnaeum Pompeium

Isocr. $=$ De Isocrate

Lys. $=$ De Lysia

Euripides, c. $480-406 / 5$ в.c.

Athenian tragedian

Alc. $=$ Alcestis

Andr. $=$ Andromache

Elect. $=$ Electra

$\mathrm{Hel} .=$ Helena

Heraclid. $=$ Heraclidae

Hipp. = Hippolytus

IA = Iphigenia Aulidensis

$I T=$ Iphigenia Taurensis

Med. $=$ Medea

Orest. $=$ Orestes

Phoen. $=$ Phoenissae

Suppl. $=$ Supplices

Tro. $=$ Troades

Eusebius of Caesarea, c. A.D. 260-340

Christian chronographer

Praep. Ev. = Praeparatio Evangelica

Eustathius, I2th century A.D.

Archbishop of Thessalonike and Homeric scholar

Galen of Pergamon, A.D. I29-c. 210

Greek medical writer

Comm. in Hipp. Epidem. = Commentarium in

Hippocratis Epidemias

Herodotus Halicarnassensis, c. 485 -c. 425 в.c.

Greek historian

Herodas [?Herondas], fl. c. 25 о в.c.

Alexandrian mime-writer 
Hes.

$$
\text { Mim. }=\text { Mimiamboi }
$$

Hesiod(us) of Ascra, fl. c. 700 в.c.

Greek epic/didactic poet

Theog. $=$ Theogony

$W D=$ Works \& Days

Hesych.

Hesychius of Alexandria, ?5th century A.D.

Lexicographer

HHAphr

Homeric Hymn to Aphrodite

HHDem

Hippocr.

Hom.

Hygin.

Hyp.

Isocr.

John Chrysost.

Homeric Hymn to Demeter

Hippocrates of Cos, ?c. 470-?400 B.c.

Greek medical writer

$$
\begin{aligned}
& \text { Aph. = Aphorisms } \\
& \text { Aër }=\text { Airs, Waters, Places } \\
& \text { Art. }=\text { De Arte }(\text { The Art [of Medicine] }) \\
& \text { Morb. Sacr }=\text { De Morbo Sacro } \\
& (\text { The Sacred Disease }) \\
& \text { Reg. }=\text { A Regimen for Health } \\
& V M=\text { De Vetere Medicina (On Ancient Medicine) }
\end{aligned}
$$

Homer(us), ?late 8th century B.c.

Greek epic poet

$$
\begin{aligned}
& \text { Il. = Iliad } \\
& \text { Od. = Odyssey }
\end{aligned}
$$

Hyginus (not C. Julius Hyginus the librarian), ?2d century A.D.

Mythographer

Astron. $=$ Astronomica

Fab. $=$ Fabulae $($ Genealogiae $)$

Hypereides, 389-322 в.c.

Athenian orator and statesman

c. Dem. $=$ contra Demosthenem

(speech Against Demosthenes)

Isocrates, $436-338$ в.c.

Athenian orator and rhetorician

$$
\text { Ep. }=\text { Epistulae }
$$

Nicocl. $=$ Ad Nicoclem

Orat. $=$ Orationes

Panegyr: $=$ Panegyricus

John [Joannes] Chrysostomus, c. A.D. 354-407

Bishop of Constantinople 
Joseph.

Jul. Obs.

Just.

Juv.

Lactant.

Livy

Lucan

Lys.

Nep.

Nicand.

Nic. Damasc.

Ovid

Oxy. Pap.
Flavius Josephus, A.D. 37/8-c. 100

Romanized Jewish historian

$$
\begin{aligned}
& \text { Af }=\text { Antiquitates fudaicae } \\
& \text { Bf }=\text { Bellum fudaicum }
\end{aligned}
$$

Julius Obsequens, 4 th $/ 5^{\text {th }}$ century A.D.

Compiler of Roman prodigies

M. Iunianus Iustinus, ? $3 \mathrm{~d}$ or 4 th century A.D.

Historical epitomator

D. Iunius Iuuenalis, c. A.D. $55^{- \text {c. }}{ }^{13} 8$

Roman satirist

$$
\text { Sat. }=\text { Saturae }
$$

L. Caelius Firmianus or Lactantius, C. A.D. $240-$ C. 320

Christian apologist

$$
I D=\text { De Ira Dei }
$$

T. Livius Patavinus, 59 B.C.-A.D. I7

Roman historian

M. Annaeus Lucanus, A.D. 39-65

Roman epic poet

$$
B C=\text { Bellum Civile }
$$

Lysias, ?459/8-c. 38 о в.с.

Athenian metic and speechwriter

Cornelius Nepos, c. IIO-24 B.c.

Roman biographer

$$
\begin{aligned}
& \text { Eum. = Eumenes } \\
& \text { Lys. = Lysander }
\end{aligned}
$$

Nicander of Colophon, fl. с. гзо в.с.

Hellenistic didactic poet

Nicolaus of Damascus, c. 64 B.C.-c. A.D. Io

Scholar and historian

Vit. Caes. Aug. = Vita Caesaris Augusti

Publius Ovidius Naso, 43 B.C.-A.D. I7/8

Roman elegiac and epic poet

$$
\begin{aligned}
& A A=\text { Ars Amatoria } \\
& \text { Am. }=\text { Amores } \\
& \text { Fast } .=\text { Fasti } \\
& \text { Met. }=\text { Metamorphoses } \\
& R A=\text { Remedia Amoris }
\end{aligned}
$$

Oxyrhynchus Papyri 
Pal.

Paus.

Palaiphatos, fl. late $4^{\text {th }}$ century в.c.

Mythographer

Пєрi å $\pi i ́ \sigma \tau \omega \nu$ (On Incredible Things)

Pausanias of Magnesia-ad-Sipylum, fl. c. A.D. 150

Greek travel-writer

Philod.

Philodemus of Gadara, c. IIO-c. 40/35 в.c.

Greek critic, epigrammatist, and philosopher

Philostr.

Pind.

L. Flavius Philostratus, c. A.D. 18o-c. 245

Second Sophistic writer

Heroic. $=$ Heroicus

Vit. Soph. = Vitae Sophistarum

(Lives of the Sophists)

Pindar(os) of Cynoscephalae (Boeotia), 5 I8-? 438 в.с.

Greek lyric poet

$$
\begin{aligned}
& \text { Isthm. = Isthmian } \\
& \text { Nem. = Nemean } \\
& \text { Ol. }=\text { Olympian } \\
& \text { Pyth. = Pythian } \\
& \text { (= the four categories of his Epinician } \\
& \text { [Victory } \text { Odes) }
\end{aligned}
$$

Plat. Plato, c. 429-347 B.c.

Athenian philosopher

$$
\begin{aligned}
& \text { Crat. }=\text { Cratylus } \\
& \text { Epin. }=\text { Epinomis } \\
& \text { Epp. }=\text { Epistulae } \\
& \text { Gorg. = Gorgias } \\
& \text { Phaedr. }=\text { Phaedrus } \\
& \text { Phileb. = Philebus } \\
& \text { Rep. }=\text { Republic } \\
& \text { Soph. = Sophist } \\
& \text { Theaet. }=\text { Theaetetus } \\
& \text { Tim. = Timaeus }
\end{aligned}
$$

Plin.

C. Plinius Secundus, c. A.D. 23-70

Roman polymath and encyclopedist

$$
\mathrm{NH}=\text { Natural History (Historia Naturalis) }
$$

Plut.

L.(?) Mestrius Plutarch(us) of Chaeronea, c. A.D. $45^{- \text {C. }} 125$

Greek essayist and biographer

$$
\text { Ag. }=\text { Agis }
$$




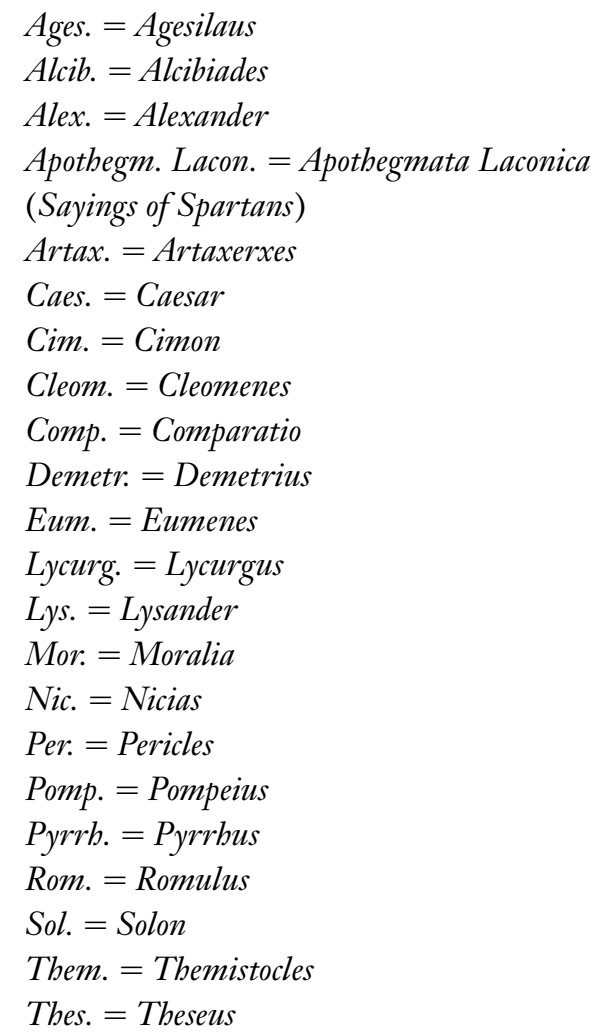

Pollux

Julius Pollux of Naucratis, 2 d cent. A.D.

Scholar and rhetorician

Onom. = Onomasticon

Polyaen. Polyaenus, 2d cent. A.D.

Military writer

Polyb. Polybius of Megalopolis, c. 200-c. II8 в.C.

Romanized Greek historian

Porph. $\quad$ Porphyry of Tyre, A.D. 234-c. 305

Scholar and philosopher

De Abst. = De Abstinentia

Ps.-Call. $\quad$ Pseudo-Callisthenes = The "Alexander Romance," dates uncertain

Ptol. Claudius Ptolemaeus of Alexandria, late $2 \mathrm{~d}$ century A.D.

Mathematician and astronomer

Tetrab. $=$ Tetrabiblos (Astrological Influences,

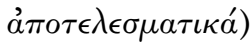


schol.

scholium or scholia

Sen.

L. Annaeus Seneca ("the Elder," E),

c. 5 O B.C. - A.D. 40

Rhetorician

L. Annaeus Seneca ("the Younger," $Y$ ),

c. 4 B.C. - A.D. 65

Man of letters, imperial minister

Controv. $=$ Controversiae $(E)$

De Prov. $=$ De Providentia $(Y)$

Ep. Mor. = Epistulae Morales $(Y)$

$N Q=$ Naturales Quaestiones $(Y)$

Sext. Emp.

Sextus Empiricus, 2d cent. A.D.

Philosopher and medical writer

Math. = Adversus Mathematicos

(Against the Professors)

Soph.

Stob.

Strabo

Sophocles, 496/5-406/5 в.с.

Athenian tragedian

$$
\begin{aligned}
& \text { El. = Electra } \\
& \text { Scyth. = Scythae }
\end{aligned}
$$

Joannes Stobaeus ("John of Stobi"), 5th century A.D.

Anthologist

Flor $=$ Florilegium

Strabo of Amaseia, c. 64 B.C.-c. A.D. 25

Romanized Greek geographer

Steph. Byz.

Stephanus of Byzantium, 5th century A.D.

Greek grammarian

Suet.

G. Suetonius Tranquillus, c. A.D. 70-c. I30

Roman imperial civil servant and biographer

Cal. $=$ Caligula

De Gramm. = De Grammaticis

Div. Aug. = Divus Augustus

Div. 7 ul. = Divus Fulius

Div. Vesp. = Divus Vespasianus

Tib. $=$ Tiberius

Tac.

Tert.

Cornelius Tacitus, c. A.D. 56-? 20

Roman historian and consular

Ann. $=$ Annales

Q. Septimius Florens Tertullianus of Carthage,

c. A.D. $160-$ c. 240

Rhetorician and theologian 
test.

Theocr.

Theophr.

Thuc.

Tzetzes

Val. Max.

Varro

Vell. Pat.

Virg.

Vitruv.

Xen.

Zenob.

Zonar.
De Anim. = De Anima

testimonium or testimonia

Theocritus of Syracuse, early 3 d century B.c.

Alexandrian pastoral poet

$$
I d .=I d y l l
$$

Theophrastus of Eresos (Lesbos), 37I-287 B.c.

Greek philosopher and botanist

$$
H P=\text { Historia Plantarum }
$$

Thucydides, c. 460-c. 395 B.c.

Athenian historian

Johannes Tzetzes, I2th century A.D.

"A copious, careless, quarrelsome Byzantine polymath" (OCD ${ }^{3}$ I568)

Chil. = Chiliades (Histories)

Valerius Maximus, early ist century A.D.

Roman anthologist

M. Terentius Varro of Reate, Ir6-27 B.c.

Roman scholar and writer

$$
R R=D e \text { Re Rustica }
$$

Velleius Paterculus, 2O/I9 B.c.-c. A.D. 4O

Roman historian

P. Vergilius Maro, 70-I9 в.с.

Roman epic and pastoral poet

$$
\begin{aligned}
& \text { Aen. = Aeneid } \\
& \text { Ecl. = Eclogues } \\
& \text { Georg. = Georgics }
\end{aligned}
$$

Vitruvius Pollio, ?50 B.c.-?A.D. 20

Roman architect and military engineer

Xenophon, c. $430-35^{\circ}$ в.c.

Athenian general and historian.

$$
\begin{aligned}
& \text { Anab. = Anabasis } \\
& \text { Hell. = Hellenica } \\
& \text { Lac. = Respublica Lacedaemoniorum } \\
& \text { Mem. = Memorabilia }
\end{aligned}
$$

Zenobius, 2d century A.D.

Sophist and paroemiographer

Johannes Zonaras, I2th century A.D.

Byzantine historian

$$
\text { Epit. = Epitome }
$$


THIS PAGE INTENTIONALLY LEFT BLANK 
FROM IKARIA TO THE STARS 
THIS PAGE INTENTIONALLY LEFT BLANK 